\title{
Local and Global Dynamics in a Discrete Time Growth Model with Nonconcave Production Function
}

\author{
Serena Brianzoni, ${ }^{1}$ Cristiana Mammana, ${ }^{2}$ \\ and Elisabetta Michetti \\ ${ }^{1}$ Dipartimento di Management, Università Politecnica delle Marche, 60121 Ancona, Italy \\ ${ }^{2}$ Dipartimento di Economia e Diritto, Università di Macerata, 62100 Macerata, Italy
}

Correspondence should be addressed to Elisabetta Michetti, michetti@unimc.it

Received 18 July 2012; Accepted 7 November 2012

Academic Editor: Juan J. Nieto

Copyright (C) 2012 Serena Brianzoni et al. This is an open access article distributed under the Creative Commons Attribution License, which permits unrestricted use, distribution, and reproduction in any medium, provided the original work is properly cited.

We study the dynamics shown by the discrete time neoclassical one-sector growth model with differential savings while assuming a nonconcave production function. We prove that complex features exhibited are related both to the structure of the coexixting attractors and to their basins. We also show that complexity emerges if the elasticity of substitution between production factors is low enough and shareholders save more than workers, confirming the results obtained while considering concave production functions.

\section{Introduction}

The standard one-sector Solow-Swan model (see [1, 2]) represents one of the most used frameworks to describe endogenous economic growth. It describes the dynamics of the growth process and the long-run evolution of the economic system.

Let $y_{t}=f\left(k_{t}\right)$ be the production function in intensive form, mapping capital per worker $k_{t}$ into output per worker $y_{t}$, then the Solow-Swan growth model describing the evolution of the state variable $k_{t}$ in a discrete time setup is given by

$$
k_{t+1}=\frac{1}{1+n}\left[(1-\delta) k_{t}+s f\left(k_{t}\right)\right]
$$

where $n \geq 0$ is the constant labor force growth rate, $\delta \in(0,1)$ is the depreciation rate of capital, and $s \in(0,1)$ is the constant saving rate. 
Most papers on economic growth considering the Solow-Swan (or neoclassical) model used the Cobb-Douglas specification of the production function, which describes a process with a constant elasticity of substitution between production factors equal to one. It is quite immediate to observe that in this formulation the system monotonically converges to the steady state (i.e., the capital per capita equilibrium) so neither cycles nor complex dynamics can be exhibited.

More recently, several contributions in the literature have considered the Constant Elasticity of Substitution (CES) production function, in order to study growth models with elasticity of substitution that can be either greater or lower than one (see for instance $[3,4]$ ). In fact, as underlined in Klump and de La Grandville [5], the elasticity of substitution between production factors plays a crucial role in the theory of economic growth since it represents one of the determinants of the economic growth level. Anyway the long run dynamics is still simple.

Another consideration is that the standard one-sector growth model does not take into account that different groups of agents (workers and shareholders) have constant but different saving propensities. Such an issue has been studied by many authors (i.e., [6-9]) in order to understand if the differential saving might influence the final dynamics of the system. In fact different but constant saving propensities make the aggregate saving propensity nonconstant and dependent on income distribution, so that multiple and unstable equilibria can occur.

Böhm and Kaas [10] investigated the following discrete-time neoclassical growth model with constant but different saving propensities between capital and labor:

$$
k_{t+1}=\frac{1}{1+n}\left[(1-\delta) k_{t}+s_{w}\left(f\left(k_{t}\right)-k_{t} f^{\prime}\left(k_{t}\right)\right)+s_{r} k_{t} f^{\prime}\left(k_{t}\right)\right]
$$

where $s_{w} \in(0,1)$ and $s_{r} \in(0,1)$ are the constant saving rates for workers and shareholders, respectively. They use a generic production function satisfying the weak Inada conditions, that is, $\lim _{k_{t} \rightarrow \infty} f\left(k_{t}\right) / k_{t}=0, \lim _{k_{t} \rightarrow 0} f\left(k_{t}\right) / k_{t}=\infty$. The authors show that instability and topological chaos can be generated in this kind of model.

Starting from Böhm and Kaas [10], recent contributions in this research line take into account other production functions in which the weak Inada conditions are not verified.

Brianzoni et al. [11-13] investigated the neoclassical growth model in discrete time with differential savings and endogenous labour force growth rate while assuming CES production function. The authors proved that multiple equilibria are likely to emerge and that complex dynamics can be exhibited if the elasticity of substitution between production factors is sufficiently low. The results obtained prove that production function elasticity of substitution plays a central role in the creation and propagation of complicated dynamics in growth models with differential saving.

As a further step in this field, Brianzoni et al. [14] firstly introduced the Variable Elasticity of Substitution (VES) production function in the form given by Revankar [15], in the discrete time neoclassical growth model (the same setup in continuous time was considered by Karagiannis et al. [16]). The use of the VES production function allows to take into account that the elasticity of substitution between production factors is influenced by the level of economic development. The authors prove that the model can exhibit unbounded endogenous growth when the elasticity of substitution between labour and capital is greater than one, as it is quite natural while the variable elasticity of substitution is assumed (and differently from CES) confirming the results obtained by Karagiannis et al. [16] in 
the continuous setup. Furthermore, the results obtained aim at confirming that the production function elasticity of substitution is responsible for the creation and propagation of complicated dynamics, as in models with explicitly dynamic optimizing behavior by the private agents (see Becker [17] for a survey about these models).

For many economic growth models based on intertemporal allocation, the hypothesis of a concave production function has played a crucial role. In fact the production function is the most important part of a growth model as it specifies the maximum output for all possible combinations of input factors and therefore determines the way the economic model evolves in time. Usually a production function is assumed to be non-negative, increasing and concave, and also to fulfill the so called Inada Conditions, that is, $f(0)=0, \lim _{k_{t} \rightarrow 0} f^{\prime}\left(k_{t}\right)=+\infty$ and $\lim _{k_{t} \rightarrow+\infty} f^{\prime}\left(k_{t}\right)=0$.

Let us focus on the meaning of condition $\lim _{k_{t} \rightarrow 0} f^{\prime}\left(k_{t}\right)=+\infty$ from an economic point of view. We take into account a region with almost no physical capital, that is there are no machines to produce goods, no infrastructure, and so forth. Then the previous condition states that it is possible to gain infinitely high returns by investing only a small amount of money. This obviously cannot be realistic since before getting returns it is necessary to create prerequisites by investing a certain amount of money. After establishing a basic structure for production, one might still get only small returns until reaching a threshold where the returns increase greatly to the point where the law of diminishing returns takes effect. In literature this fact is known as poverty traps. In other words, we should expect that there is a critical level of physical capital having the property that, if the initial value of physical capital is smaller than such a level, then the dynamic of physical capital will descend to the zero level, thus eliminating any possibility of economic growth. Thus we introduce the before mentioned threshold $\bar{k}$ in order to consider what happens to the Solow model if we do not assume a concave production function. In fact concavity assumptions provide a good approximation of a high level of economic development but is not always applicable to lessdeveloped countries. A small amount of money may have an effect in the short run but this effect will tend to zero in the long run if there are no more investments. Thus it makes sense to assume that only an amount of money larger than some threshold will lead to returns.

The first model with nonconcave production function was introduced by Clark [18] and Skiba [19]. Following such works several contributions have then focused on the existence and implications of critical levels (see, among others, [20-22]). Capasso et al. [23] focused on a parametric class of nonconcave production functions which can be considered as an extension of the standard Cobb-Douglas production function; the authors study the Solow growth model in continuous time and show the existence of rich dynamics by mainly using numerical techniques.

In the present work we study the discrete time one sector Solow-Swan growth model with differential savings as in Böhm and Kaas [10], while assuming that the technology is described by a nonconcave production function in the form given by Capasso et al. [23]. Our main goal is to describe the qualitative and quantitative long run dynamics of the growth model to show that complex features can be observed and to compare the results obtained with the ones reached while considering the CES or the VES technology.

The results of our analysis show that our model can exhibit complexity related both to the structure of the attractors of the system (passing from locally stable fixed points to bounded fluctuations or, even, to chaotic patterns), to the coexistence of attractors giving rise to multistability phenomenon and, finally, to the structure of the basins of attraction (from a simple connected to a non-connected one). 
The role of the production function elasticity of substitution has been related to the creation and propagation of complicated dynamics. In fact, similarly to what happens with the CES and VES production function (see Brianzoni et al. [11, 13, 14]), if shareholders save more than workers and the elasticity of substitution between production factors is low, then fluctuations may arise.

The paper is organized as follows. In Section 2 we introduce the model. In Section 3 we perform the dynamic analysis. In Section 4 we deal with the case in which complex dynamics are exhibited. Section 5 concludes our paper.

\section{The Model}

Let $y_{t}=f\left(k_{t}\right)$ be the production function in intensive form, mapping capital per worker $k_{t}$ into output per worker $y_{t}$, then, the neoclassical one-sector growth model with different but constant saving rates between worker and shareholders as proposed by Böhm and Kaas [10] is given by:

$$
k_{t+1}=\frac{1}{1+n}\left[(1-\delta) k_{t}+s_{w} w\left(k_{t}\right)+s_{r} k_{t} f^{\prime}\left(k_{t}\right)\right]
$$

where $\delta \in(0,1)$ is the depreciation rate of capital, $s_{w} \in(0,1)$ and $s_{r} \in(0,1)$ are the constant saving rates for workers and shareholders, respectively, and $n \geq 0$ is the constant labor force growth rate.

The wage rate $w\left(k_{t}\right)$ must equal the marginal product of labor, that is, $w\left(k_{t}\right):=f\left(k_{t}\right)-$ $k_{t} f^{\prime}\left(k_{t}\right)$, furthermore shareholders receive the marginal product of capital $f^{\prime}\left(k_{t}\right)$ hence the total capital income per worker is $k_{t} f^{\prime}\left(k_{t}\right)$, then (2.1) may be re-written as follows:

$$
k_{t+1}=\frac{1}{1+n}\left[(1-\delta) k_{t}+s_{w} f\left(k_{t}\right)+\left(s_{r}-s_{w}\right) k_{t} f^{\prime}\left(k_{t}\right)\right]
$$

In order to obtain the dynamic system describing the evolution of the capital per capita as given by (2.2) we have to specify the production function.

Economic growth models used to consider the hypothesis of a production function satisfing the following standard economic properties: $f(k)>0, f^{\prime}(k)>0$, and $f^{\prime \prime}(k)<0$, for all $k>0$; observe that such properties hold for the Cobb-Douglas, CES, and VES production functions. In addition both the VES and the Cobb-Douglas production functions verify one of the Inada Conditions, that is $\lim _{k_{t} \rightarrow 0} f^{\prime}\left(k_{t}\right)=+\infty$.

According to the previous condition an economy with no physical capital can gain infinitely high returns by investing only a small amount of money, hence it cannot be considered a realistic assumption. In fact, it is quite obvious to assume that a certain amount of investment is needed before reaching a threshold capital level $\bar{k}$ such that great returns are obtained only for $k_{t} \geq \bar{k}$. To be more precise, a more realistic economic assumption might take into account that a $\bar{k}>0$ does exist such that the production function $f(k)$ is characterized by increasing (decreasing) marginal returns for $k_{t}<\bar{k}\left(k_{t} \geq \bar{k}\right)$. This fact is known as the poverty trap as, if the initial value of capital is sufficiently small, then the dynamic law of capital accumulation will push the capital level to zero and no economic growth will take place. Given such considerations, the concavity assumption provides a good approximation for 
a production function only at high level of economic development while, if a less developed country is considered, then a nonconcave production function has to be taken into account.

Following Capasso et al. [23], we consider a sigmoidal production function (i.e., it shows an S-shaped behavior) given by

$$
f\left(k_{t}\right)=\frac{\alpha k_{t}^{p}}{1+\beta k_{t}^{p}},
$$

where $\alpha>0, \beta>0$, and $p \geq 2$. Observe that

$$
\begin{gathered}
f^{\prime}\left(k_{t}\right)=\frac{\alpha p k_{t}^{p-1}}{\left(1+\beta k_{t}^{p}\right)^{2}}, \\
f^{\prime \prime}\left(k_{t}\right)=\frac{\left(p \alpha k_{t}^{p-2}\right)\left[p\left(1-\beta k_{t}^{p}\right)-\left(1+\beta k_{t}^{p}\right)\right]}{\left(1+\beta k_{t}^{p}\right)^{3}},
\end{gathered}
$$

and recall that the Inada Conditions are $f(0)=0, \lim _{k_{t} \rightarrow 0} f^{\prime}\left(k_{t}\right)=+\infty$ and $\lim _{k_{t} \rightarrow+\infty} f^{\prime}\left(k_{t}\right)=0$. Then function (2.3) is positive for all $k_{t}>0$, strictly increasing and it is a convex-concave production function. In fact $f^{\prime}\left(k_{t}\right)>0$, for all $k_{t}>0$ while a $\bar{k}>0$ exists such that $f^{\prime \prime}\left(k_{t}\right)>(<) 0$, if $0<k_{t}<\bar{k}\left(k_{t}>\bar{k}\right)$, being $\bar{k}=((p-1) / \beta(p+1))^{1 / p}$ the inflection point of $f$. Furthermore the production function (2.3) does not satisfy one of the Inada Conditions, in fact

$$
\lim _{k_{t} \rightarrow 0} f^{\prime}\left(k_{t}\right)=\lim _{k_{t} \rightarrow 0} \frac{\alpha p k_{t}^{p-1}}{\left(1+\beta k_{t}^{p}\right)^{2}}=0 .
$$

Observe also that the elasticity of substitution between production factors of function (2.3) depends on the level of the capital per-capita $k_{t}$ as it is given by

$$
\sigma(k)=1+\frac{\beta p k^{p}}{p\left(1-\beta k^{p}\right)-\left(1+\beta k^{p}\right)}
$$

so that also this function belongs to the class of VES production functions, that is, the elasticity of substitution depends on $k$. Observe the role played by the constant $p$ : if $p$ is great enough then $\sigma(k)$ decreases w.r.t. $p$ where

$$
\sigma_{p}^{\prime}(k)=\frac{\beta k^{p}\left[p^{2} \ln k-p \ln k-1-\beta k^{p}\right]}{\left[p-1-\beta k^{p}(p+1)\right]^{2}}
$$

and

(i) if $k>1$ then $\lim _{p \rightarrow+\infty} \sigma(k)=0$;

(ii) if $k<1$ then $\lim _{p \rightarrow+\infty} \sigma(k)=1$;

(iii) if $k=1$ then $\lim _{p \rightarrow+\infty} \sigma(k)=1 /(1-\beta)$. 
By substituting (2.3) and (2.4) into (2.2) we obtain the following one dimensional map describing the capital accumulation:

$$
k_{t+1}=F\left(k_{t}\right)=\frac{1}{1+n}\left[(1-\delta) k_{t}+\frac{\alpha k_{t}^{p}}{1+\beta k_{t}^{p}}\left(s_{w}+p \frac{s_{r}-s_{w}}{1+\beta k_{t}^{p}}\right)\right]
$$

with $F$ continuous and smooth function for all $k_{t}>0$.

\section{Stability of Steady States}

We first consider the question of the existence and number of fixed points or steady states of map (2.8) and then we discuss about their stability depending on all the parameter values.

The estabilishment of the number of steady states is not trivial to solve, considering the high variety of parameters. As a general result, the map $F$ always admits one fixed point characterized by zero capital per capita, that is, $k=0$ is a fixed point for any choice of parameter values. Anyway steady states which are economically interesting are those characterized by positive capital per worker.

In order to determine the positive fixed points of $F$, let us define the following function:

$$
G\left(k_{t}\right):=\frac{k_{t}^{p-1}}{1+\beta k_{t}^{p}}\left(s_{w}+p \frac{s_{r}-s_{w}}{1+\beta k_{t}^{p}}\right), \quad k_{t}>0,
$$

then solutions of $G\left(k_{t}\right)=(n+\delta) / \alpha$ are positive fixed points of $F$. The following proposition states the number of fixed points of $F$ according to the parameter values.

Proposition 3.1. Let $F$ be given by (2.8) and $s_{r}>s_{w}$. Then a $k_{0}>0$ does exist such that:

(i) if $(n+\delta) / \alpha>G\left(k_{0}\right)$, F has a unique fixed point given by $k_{t}=0$;

(ii) if $(n+\delta) / \alpha=G\left(k_{0}\right), F$ has two fixed points given by $k_{t}=0$ and $k_{t}=k^{*}>0$;

(iii) if $(n+\delta) / \alpha<G\left(k_{0}\right)$, F has three fixed points given by $k_{t}=0$ and $0<k_{1}<k_{2}$.

Proof. $k_{t}=0$ is a solution of $k_{t}=F\left(k_{t}\right)$ for all parameter values hence it is a fixed point. Function (3.1) is such that $G\left(k_{t}\right)>0$, for all $k_{t}>0$, furthermore $\lim _{k_{t} \rightarrow 0^{+}} G\left(k_{t}\right)=0$ and $\lim _{k_{t} \rightarrow+\infty} G\left(k_{t}\right)=0$. Hence $G\left(k_{t}\right)$ has at least one critical point being a maximum point (namely $k_{0}$ ). We prove that it is unique.

Let $G(k):=G\left(k_{t}\right)$ then, given the production function $f$ as defined in (2.3), we obtain

$$
G(k)=\frac{f}{\alpha k}\left(s_{w}+p \frac{s_{r}-s_{w}}{1+\beta k^{p}}\right) .
$$

Define $\Delta s=s_{r}-s_{w}$ and $M(k)=\beta k^{p}$ then we obtain

$$
G^{\prime}=\frac{1}{\alpha}\left[\frac{f^{\prime} k-f}{k^{2}}\left(s_{w}+p \frac{\Delta s}{1+M}\right)-\frac{f}{k}\left(\frac{p^{2} \Delta s M}{k(1+M)^{2}}\right)\right] .
$$


Making use of relation $f^{\prime}=p f / k(1+M)$ we reach the following expression:

$$
G^{\prime}=\frac{f}{\alpha(1+M)^{2} k^{2}}\left[a M^{2}+b M+c\right],
$$

where $a=-s_{w}, b=s_{w}(p-2)-\Delta s\left(p^{2}+p\right)$ and $c=s_{w}(p-1)+\Delta s\left(p^{2}-p\right)$.

The critical points of $G$ are the positive solutions of $a M^{2}+b M+c=0$; being $a<0$ and $c>0$ then it admits a unique positive solution given by $\bar{M}=\left(-b-\sqrt{b^{2}-4 a c}\right) / 2 a>0$. Hence function $G$ has a unique maximum point $k_{0}=(\bar{M} / \beta)^{1 / p}$ and consequently it intersects the positive and constant function $g=(n+\delta) / \alpha$ in two, one, or no points if $g<G\left(k_{0}\right), g=G\left(k_{0}\right)$ or $g>G\left(k_{0}\right)$, respectively.

According to the previous proposition, if $s_{r}>s_{w}$ (i.e., shareholders save more than workers) the map always admits the equilibrium $k=0$, moreover up to two additional (positive) fixed points can exist according to the parameter values hence multiple equilibria are exhibited. We now wish to study the local stability of these equilibria. Let us consider first the origin.

Proposition 3.2. Let $F$ be as given in (2.8) and $s_{r}>s_{w}$. Then the equilibrium $k=0$ is always locally stable.

Proof. Firstly notice that function $F$ may be written in terms of function $G$ being

$$
F(k)=\frac{1}{1+n}[(1-\delta) k+\alpha k G(k)]
$$

hence $F^{\prime}(k)=(1 /(1+n))\left[1-\delta+\alpha\left(G(k)+k G^{\prime}(k)\right)\right]$. Being $\lim _{k \rightarrow 0^{+}} G(k)=0$ and $\lim _{k \rightarrow 0^{+}} k G^{\prime}(k)=$ 0 , then $F^{\prime}(0)=((1-\delta) /(1+n)) \in(0,1)$ and consequently the origin is a locally stable fixed point for map $F$.

Observe that as in Capasso et al. [23] the use of the S-shaped production function implies the existence of a poverty trap. Recall that in models previously proposed in which production function is concave (as the CES production function in Brianzoni et al. [11,13] or the VES production function in Brianzoni et al. [14]) the origin is always a locally unstable fixed point hence the economy will converge in the long run to positive level growth rates (eventually with periodic or even $a$-periodic dynamic features). Differently, in our new setup, the origin is always locally stable hence economies starting from a sufficiently low level of capital per-capita may be captured by a poverty trap. More precisely, there exists a critical level of physical capital having the property that, if the initial value of physical capital is smaller than such a level, then the dynamic of physical capital will converge to zero, thus eliminating any possibility of economic growth.

Furthermore, from the previous proposition it follows that the fixed point $k=0$ is globally stable when it is the unique steady state owned by the map (i.e., case (i) of Proposition 3.1), see Figures 1 and 2(a). According to Proposition 3.1, this happens when $(n+\delta) / \alpha>G\left(k_{0}\right)$.

Since $G\left(k_{0}\right)$ does not depend on $\alpha$ and being $\alpha>0$ we can conclude that $\exists \bar{\alpha}$ such that $k=0$ is the unique steady state owned by the system for all $0<\alpha<\bar{\alpha}$ (this means that the production function upper bound is small enough). Similarly, being $n$ upper unbounded, 


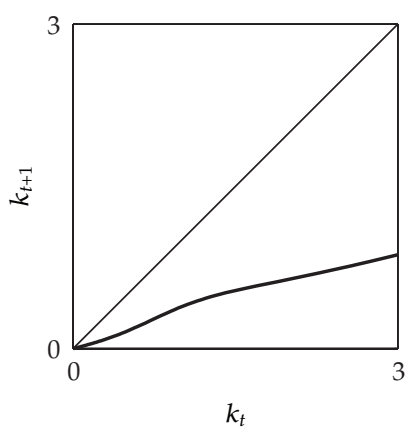

(a)

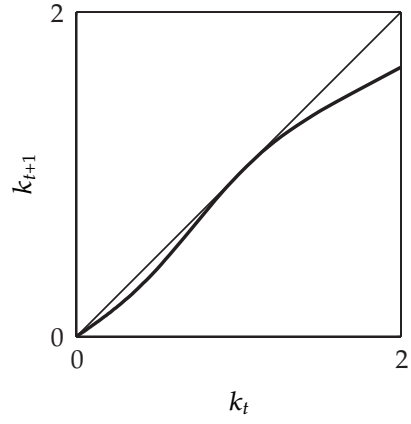

(b)

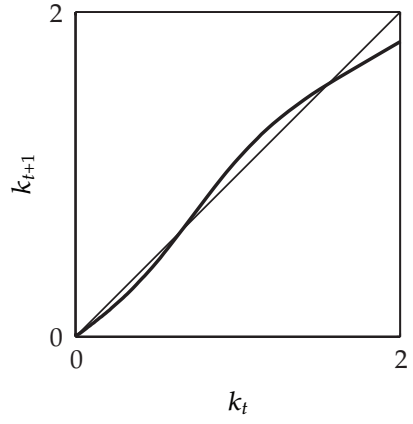

(c)

Figure 1: Map $F$ and its fixed points in the case of strict monotonicity for the following parameter values: $\beta=0.5, s_{w}=0.2, s_{r}=1, p=3, \delta=0.2, \alpha=0.3$. In (b) $n=\alpha G\left(k_{0}\right)-\delta=0.1606=\bar{n}$; in (a) $n>\bar{n}$, in (c) $n<\bar{n}$.

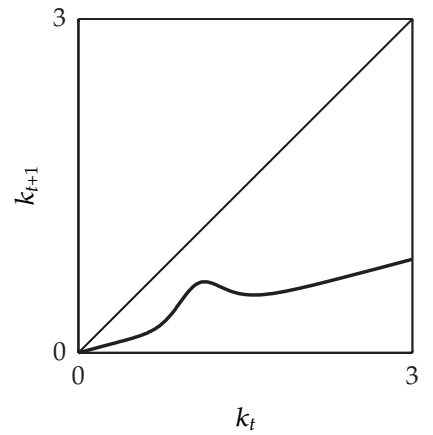

(a)

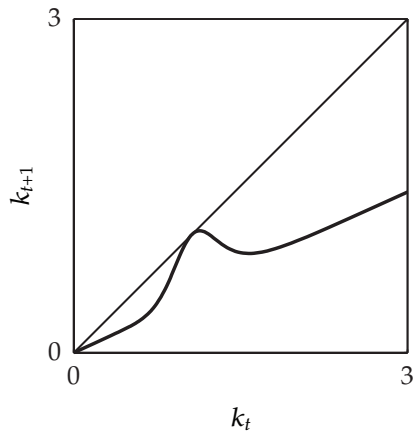

(b)

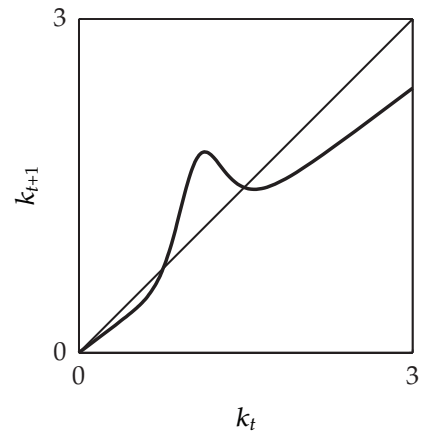

(c)

Figure 2: Map $F$ and its fixed points in the case of non-invertibility for the following parameter values: $\beta=0.5, s_{w}=0.2, s_{r}=1, p=8, \delta=0.2, \alpha=0.3$. In (b) $n=\bar{n}=0.7444$; in (a) $n>\bar{n}$, in (c) $n<\bar{n}$.

then a $\bar{n}$ does exist such that $k=0$ is the unique steady state of the model for all $n>\bar{n}$, (i.e., if population growth rate is sufficiently high) given the other parameter values. In such cases the poverty trap cannot be avoided and the system will converge to a zero growth rate.

We now focus on the case stated in Proposition 3.1(iii), that is, map $F$ has three fixed points given by $k_{t}=0, k_{1}>0$ and $k_{2}>0$, being $k_{1}<k_{2}$. About the local stability of the two positive fixed points, we start proving the following proposition.

Proposition 3.3. Let $F$ be as given in (2.8), $s_{r}>s_{w}$, and $(n+\delta) / \alpha<G\left(k_{0}\right)$. Then the equilibrium $k_{1}$ is always locally unstable.

Proof. Recall that

$$
F(k)=\frac{1}{1+n}[(1-\delta) k+\alpha k G(k)]
$$

hence $F^{\prime}\left(k_{1}\right)=(((1-\delta) /(1+n))+(\alpha /(1+n)))\left[\left(G\left(k_{1}\right)+k_{1} G^{\prime}\left(k_{1}\right)\right)\right]$. From Proposition 3.1 it follows that $G\left(k_{1}\right)=(n+\delta) / \alpha$, hence, by substituting in the previous formula, we obtain that 
$F^{\prime}\left(k_{1}\right)=1+(\alpha /(1+n)) k_{1} G^{\prime}\left(k_{1}\right)>1$ since $G^{\prime}\left(k_{1}\right)>0$ (in fact $k_{1}$ is located on the increasing branch of function $G)$.

From the previous proposition it follows that $k_{1}$ is a locally unstable fixed point; furthermore map $F$ is increasing in such a point. Let us now focus on the local stability of $k_{2}$ by proving the following proposition.

Proposition 3.4. Let $F$ be as given in (2.8), $s_{r}>s_{w}$, and $(n+\delta) / \alpha<G\left(k_{0}\right)$. Then $F^{\prime}\left(k_{2}\right)<1$.

Proof. Similarly to the considerations used to prove Proposition 3.3 we obtain that $F^{\prime}\left(k_{2}\right)=$ $1+(\alpha /(1+n)) k_{2} G^{\prime}\left(k_{2}\right)<1$ being $G^{\prime}\left(k_{2}\right)<0$ as $k_{2}$ is located on the decreasing branch of function $G$.

As a consequence, the steady state $k_{2}$ may be locally stable if $F^{\prime}\left(k_{2}\right) \in(-1,1)$ or locally unstable if $F^{\prime}\left(k_{2}\right)<-1$; in this last case $k_{2}$ loses its stability via flip bifurcation and a two period-cycle may be created proving that our model may produce fluctuations in economic growth (we will explain the route to chaos in the following section).

In order to obtain results concerning the local stability of $k_{2}$ we use the following arguments to prove that function $F$ may be strictly increasing or bimodal, according to the parameter values.

Recall that $F$ may be written in terms of $G$, and consider the expression for $F^{\prime}$ as given in the proof of Proposition 3.2, that is,

$$
F^{\prime}(k)=\frac{1}{1+n}\left[1-\delta+\alpha\left(G(k)+k G^{\prime}(k)\right)\right]
$$

then $F^{\prime}=0$ if and only if $G(k)+k G^{\prime}(k)=(\delta-1) / \alpha<0$. Observe that $G(k)+k G^{\prime}(k)$ can be written in terms of $M(k)=\beta k^{p} \geq 0$ (being $M$ a continuous, differentiable and strictly increasing function of $k$ ) as follows:

$$
G(k)+k G^{\prime}(k)=\frac{p M^{(p-1) / p}}{\beta^{(p-1) / p}(1+M)^{3}}\left[\left(s_{w}-p \Delta s\right) M+s_{w}+p \Delta s\right]=H(M(k)),
$$

hence the critical points of $F$ are solutions of

$$
H(M(k))=(\delta-1) / \alpha
$$

Observe that if $s_{w}-p \Delta s \geq 0$ then $H(M(k))$ is positive for all $k>0$ and consequently (3.9) has no solution. In such a case $F$ is strictly increasing and no complicated dynamics occurs.

In order to obtain a sufficient condition for $F$ being non-invertible, we study function $H(M)$ to determine the number of solutions of (3.9).

Assume $s_{w}-p \Delta s<0$. Function $H(M)$ is such that $H(0)=0, \lim _{M \rightarrow \infty} H(M)=0$ and $H(M)>(<) 0$ if $M<(>)\left(s_{w}+p \Delta s\right) /\left(-s_{w}+p \Delta s\right)$ being $\left(s_{w}+p \Delta s\right) /\left(-s_{w}+p \Delta s\right)>1$. As a consequence $H(M)$ can intersect the constant and negative function $h=(\delta-1) / \alpha$ only in points belonging to the interval $I=\left(\left(s_{w}+p \Delta s\right) /\left(-s_{w}+p \Delta s\right),+\infty\right)$. Obviously $H$ admits at least one maximum point $M_{M}$ less than $\left(s_{w}+p \Delta s\right) /\left(-s_{w}+p \Delta s\right)$ and at least one minimum point $M_{m}$ belonging to the interval $I$. We now want to prove that $H$ admits only 
one minimum point $M_{m}$, so that it may intersect function $h$ at most in two points $M_{1}$ and $M_{2}$ (in such a case funtion $F$ is bimodal, that is it admits both a local maximum and a local minimum point).

We first compute the derivative of function $H$. We rewrite function $H$ as follows:

$$
H(M)=\frac{A M^{2}+B M}{M^{1 / p}(1+M)^{3}}
$$

where $A=\left(p / \beta^{(p-1) / p}\right)\left(s_{w}-p \Delta s\right)<0$ and $B=\left(p / \beta^{(p-1) / p}\right)\left(s_{w}+p \Delta s\right)>0$, hence

$$
H^{\prime}(M)=\frac{1}{p M^{1 / p}(1+M)^{4}}\left[(-1-p) A M^{2}+[(2 p-1) A-(2 p+1) B] M+(p-1) B\right]
$$

so that it admits two positive zeroes that are $M_{M}$ and $M_{m}$, respectively, where

$$
M_{m}=\frac{-[(2 p-1) A-(2 p+1) B]+\sqrt{[(2 p-1) A-(2 p+1) B]^{2}+4\left(p^{2}-1\right) A B}}{2(-1-p) A}
$$

and consequently if $H\left(M_{m}\right)<(\delta-1) / \alpha$ then (3.9) has two solutions $\left(M_{1}\right.$ and $\left.M_{2}\right)$. In such a case function $F$ is bimodal hence $k_{M}=\left(M_{1} / \beta\right)^{1 / p}$ and $k_{m}=\left(M_{2} / \beta\right)^{1 / p}$ are, respectively, its maximum and minimum points.

The previous considerations prove the following proposition.

Proposition 3.5. Let $F$ be as given in (2.8), $s_{r}>s_{w}$.

(i) Let $s_{w}-p \Delta s \geq 0$, then map $F$ is strictly increasing.

(ii) Let $s_{w}-p \Delta s<0$ and define $A=\left(p / \beta^{(p-1) / p}\right)\left(s_{w}-p \Delta s\right)<0, B=\left(p / \beta^{(p-1) / p}\right)\left(s_{w}+\right.$ $p \Delta s)>0$ and $H(M)=\left(A M^{2}+B M\right) / M^{1 / p}(1+M)^{3}$. Then $M_{m}>1$ does exist such that:

(a) if $H\left(M_{m}\right) \geq(>)(\delta-1) / \alpha$ then $F$ is (strictly) increasing;

(b) if $H\left(M_{m}\right)<(\delta-1) / \alpha$ then $F$ admits a maximum point $k_{M}$ and a minimum point $k_{m}$ such that $1<k_{M}<k_{m}$.

We first focus on case (i) of Proposition 3.5 stating a sufficient condition such that the map is increasing (see Figure 1). Obviously in such a case, if the origin is the unique fixed point, then it is globally stable (case (i) of Proposition 3.1).

When $G\left(k_{0}\right)$ crosses the value $(n+\delta) / \alpha$ then a fold bifurcation occurs creating a new fixed point namely $k^{\star}$ (case (ii) of Proposition 3.1). Such a steady state is a non-iperbolic fixed point being its eigenvalue equal to one. Anyway, as $F$ is strictly increasing, then $k^{\star}$ attracts trajectories having initial condition (i.c.) $k_{0} \geq k^{\star}$ while the interval $\left[0, k^{\star}\right)$ is the basin of attraction of the origin. Observe that in such a case the poverty trap can be avoided if the economy starts from a sufficiently high level of capital per capita. 
Finally, in case (iii) of Proposition 3.1 three fixed points are owned by F; furthermore $F^{\prime}\left(k_{2}\right) \geq 0$. Hence, taking into account the result proved in Proposition 3.4, it must be $F^{\prime}\left(k_{2}\right) \in(0,1)$ (see Figure $1(\mathrm{c})$ ) and consequently the steady state $k_{2}$ is locally stable. The unstable fixed point $k_{1}$ separates the basin of attraction of the origin and that of $k_{2}$. In this case both the structure of the attractors (fixed points) and that of their basins (connected sets) are simple.

In all the above mentioned cases only simple dynamics is presented: that is, the economic system monotonically converges to a steady state characterized by a zero (poverty trap) or a positive capital per capita growth rate. Observe that, according to condition (i) of Proposition 3.5 a $\bar{\Delta} s$ does exist such that $F$ is increasing for all $\Delta s<\bar{\Delta} s$. This means that no cycles or complex features are observed if the difference between the two propensities to save is low enough, confirming what proved in Brianzoni et al. $[11,13,14]$ in which concave production functions were taken into account (the CES and the VES function, resp.).

In order to find more complex long run growth patterns we have to focus on case (ii) of Proposition 3.5 stating conditions such that $F$ is non-invertible (see Figure 2).

First notice that if $F$ has only the origin as a fixed point then, as in the previous case, it is globally stable (case (i) of Proposition 3.1). Hence we want to consider the case in which more than one fixed point is owned by $F$, that is, condition (ii) or (iii) of Proposition 3.1 holds.

Observe that, for any given value of the other parameters, if $\alpha$ is great enough then $(n+\delta) / \alpha \leq G\left(k_{0}\right)$, hence $F$ has two or three fixed points: multiple equilibria exist and their basins of attraction have to be discussed. Furthermore, since $F$ can be bimodal, then complex dynamics can be exhibited, so that the role of the parameters of the model and the route to complexity must be described.

In fact the iterated application of a noninvertible map repeatedly folds the state space allowing to define a bounded region where asymptotic dynamics are trapped. Furthermore, the iterated application of the inverses repeatedly unfolds the state space, so that a neighborhood of an attractor may have preimages far from it. This may give rise to complexity both in the qualitative structure of the attractor (that can be periodic or chaotic) and in the topological structures of the basins (that can even be formed by the union of several disjoint portions).

Condition $s_{w}-p \Delta s<0$ in Proposition 3.5(ii) is necessary for $F$ being bimodal. In such a case $\lim _{p \rightarrow+\infty} H\left(M_{m}\right)=-\infty$ so that a $p_{1}>0$ does exist such that $F$ is bimodal for all $p>p_{1}$. Being the non-invertibility of $F$ a necessary condition for our model having complex dynamics, the previous condition is necessary for cycles of chaotic dynamics to be observed. In addition another necessary condition for our setup showing nontrivial dynamics is that three fixed points are owned by $F$ that is $(n+\delta) / \alpha<G\left(k_{0}\right)$. Similarly to the previous consideration, we observe that $\lim _{p \rightarrow+\infty} G\left(k_{0}\right)=+\infty$ and consequently a $p_{2}>0$ does exist such that $F$ admits three fixed points for all $p>p_{2}$. Let $\bar{p}=\max \left\{p_{1}, p_{2}, 2\right\}$, then the following proposition trivially holds.

Proposition 3.6. Let $F$ be as given in (2.8), $s_{r}>s_{w}$. Then a $\bar{p}>0$ does exists such that $F$ is bimodal and admits three fixed points for all $p>\bar{p}$.

The previous condition is necessary for cycles or chaos to be observed in our model. It is straightforward to observe that complex features can emerge if $p$ is great enough as long as $s_{r}>s_{w}$. This fact proves that both the elasticity of substitution and the different saving propensities contribute to generate complexity in economic growth, as in models with concave production functions. 


\section{Complex Dynamics}

We now want to study the qualitative asymptotic properties of the sequence generated by $F$ in the case of non-invertibility, by combining analytical tools and numerical simulations.

We will prove that a generic trajectory may converge to a given steady state or to a more complex attractor, that may be periodic (an $m$-period cycle) or chaotic. In this last case we will determine the bounded set of the line where the system's dynamics are trapped and we will describe the complexity of the attractors belonging to these sets.

Furthermore, we will prove that multistability, that is, the existence of many coexisting attractors (that may be periodic or even chaotic sets) emerges.

Finally, as our map is characterized by coexisting attractors, we will study global bifurcations occurring as some parameters are varied that are responsible for changing in the properties of the attracting sets and of their basins of attraction (that may consist of infinitely many unconnected sets).

These problems lead to different routes to complexity, one related to the complexity of the attracting sets which characterize the long run time evolution of the dynamic process, the other one related to the complexity of the boundaries which separate the basins when several coexisting attractors are present. These two different kinds of complexity are not related in general, in the sense that very complex attractors may have simple basin boundaries, whereas boundaries which separate the basins of simple attractors, such as coexisting stable equilibria, may have very complex structures.

\subsection{Multistability and Complex Attractors}

Recall that function $F$ always admits a locally stable fixed point given by $k=0$, hence the set $A_{0}=\{0\}$ is an attractor for $F$ for all parameter values.

In order to assess the possibility of complex dynamics arising, we have to consider the case in which Proposition 3.6 holds, that is $F$ is bimodal (it has two critical points $k_{M}$ and $k_{m}$ ) and admits three fixed points. Let us start considering the stability of the fixed point $k_{2}$ and the bifurcations it undergoes as some parameters vary. As we pointed out, the eigenvalue associated to $k_{2}$ can be positive or negative depending on the position of $k_{2}$ w.r.t. the critical points, more precisely $F^{\prime}\left(k_{2}\right)<0$ if and only if $k_{2} \in\left(k_{M}, k_{m}\right)$. Obviously if $k_{2} \leq k_{M}$ or $k_{2} \geq k_{m}$ no complex dynamics can arise.

Let $p>\bar{p}$, then after the fold bifurcation occurring at $(n+\delta) / \alpha=G\left(k_{0}\right)$ (see Proposition 3.1), two fixed points $k_{1}$ and $k_{2}$ are created (for instance when $n$ decreases crossing a given value $\bar{n}$ or $\alpha$ increases crossing a given value $\bar{\alpha}$ ). Immediately after this bifurcation the two fixed points born from a little perturbation of the parameters both belong to the increasing branch of $F$ (as it is continuous and differentiable also w.r.t. the parameters of the model), hence $0<k_{1}<k_{2}<k_{M}<k_{m}$. As a consequence $k_{1}$ is unstable while the other fixed points are (locally) stable.

Let $F\left(k_{m}\right)>k_{1}$, then the unstable fixed point $k_{1}$ separates the basin of attraction of $A_{0}$ given by $B\left(A_{0}\right)=\left[0, k_{1}\right)$ from the basin of attraction of the positive steady state $k_{2}$ given by $B\left(k_{2}\right)=\left(k_{1},+\infty\right)$. In this situation an economic policy trying to increase investment can be able to push the economy out of the poverty trap toward a positive long run economic growth rate.

In the case previously described $k_{2}$ is a locally stable fixed point. Anyway, as underlined, $k_{2}$ may be locally unstable and in such a case a more complex attractor may appear around $k_{2}$; we call such an attractor $A$. Obviously, given the shape of map $F$, a necessary 


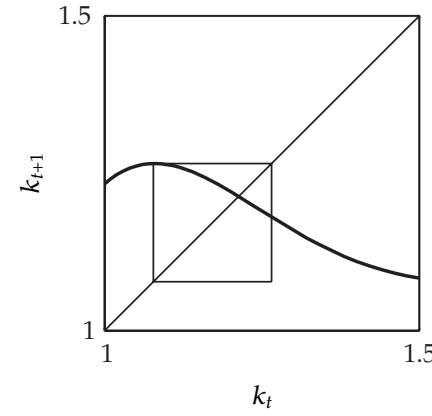

(a)

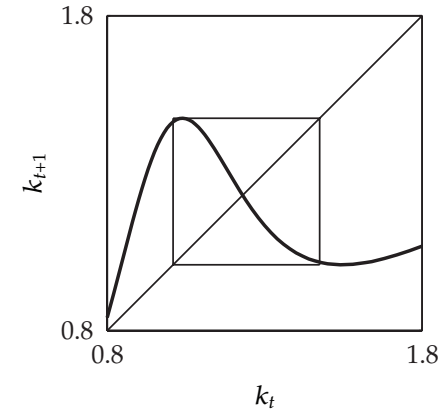

(b)

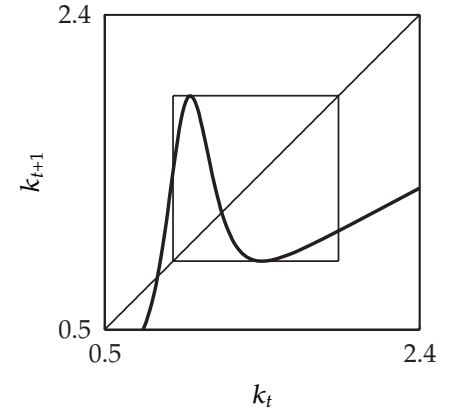

(c)

Figure 3: Map $F$ and set $S$ for $\beta=0.9, \alpha=1, n=0.5, s_{w}=0.1$ and $s_{r}=0.7$. (a) $p=6$ and $F_{S}$ is strictly decreasing; (b) $p=8$ and $F_{S}$ is unimodal (the maximum point belongs to $S$ ); (c) $p=12$ and $F_{S}$ is bimodal (both $k_{M}$ and $k_{m}$ belong to $S$ ).

condition for $A$ having a complicated structure is $k_{M}<k_{2}<k_{m}$ (so that the fixed point $k_{2}$ belongs to the decreasing branch of $F$ ). Given the analytical form of $F$ we cannot give conditions for such a case, anyway we can describe the transition to chaos by means of numerical simulations. In this subsection we want to describe the structure of $A$ by focusing on the case in which $F\left(k_{m}\right)>k_{1}$. A bimodal map having a similar behaviour to the one we are studing is in Liz [24].

Being $F$ bimodal it admits a forward invariant interval bounded by the local maximum and the local minimum. The following proposition states the existence of a trapping set for the map $F$ for economically meaningful parameter range related to nontrivial dynamics.

Proposition 4.1. Let $F$ be as given in (2.8), $s_{r}>s_{w}, p>\bar{p}$ and $F\left(k_{m}\right)>k_{1}$. If $k_{M}<k_{2}<k_{m}$ then the set $S=\left[F\left(k_{m}\right), F\left(k_{M}\right)\right]$ is trapping.

In fact $F$ acts from the interval $S$ into itself, in other words $S$ is a closed region positively invariant. Recall that set $E \subseteq \mathbb{R}_{+}$is positively (negatively) invariant if $F^{t}(E) \subseteq E$ $\left(F^{t}(E) \supseteq E\right)$, for all $t \in \mathbb{Z}_{+}$. $E$ is called invariant when it is both positively and negatively invariant. Moreover any trajectory generated from an i.c. in $\left(k_{1},+\infty\right)$ enters in $S$ after a finite number of iterations, that is, $S$ is absorbing. As $S$ is trapping then the attractor $A$ (different from $A_{0}$ ) of $F$ must belong to $S$. Obviously it may consists of a fixed point $\left(k_{2}\right)$ or a more complex set or two coexisting attractors may be owned. Anyway, observe that the only way in which $k_{2}$ may lose stability is via period-doubling bifurcation.

According to the previous proposition every i.c. $k_{0}>k_{1}$ generates bounded trajectories converging to an attractor included into the trapping interval $S$.

As $S$ is trapping, we can restrict map $F$ to such a set; we call $F_{S}$ the map $F$ defined in $S$ and we describe the properties of $F_{S}$. Observe that the following cases may occur (see Figure 3).

(i) If $F\left(k_{M}\right)<k_{m}$ and $F\left(k_{m}\right)>k_{M}$ then $k_{M} \notin S$ and $k_{m} \notin S$ (we are considering the case in which the critical points do not belong to $S$ ). In such a case $F_{S}$ is strictly decreasing and $A$ consists of a fixed point or of a 2-period cycle; more complex dynamics are ruled out. 


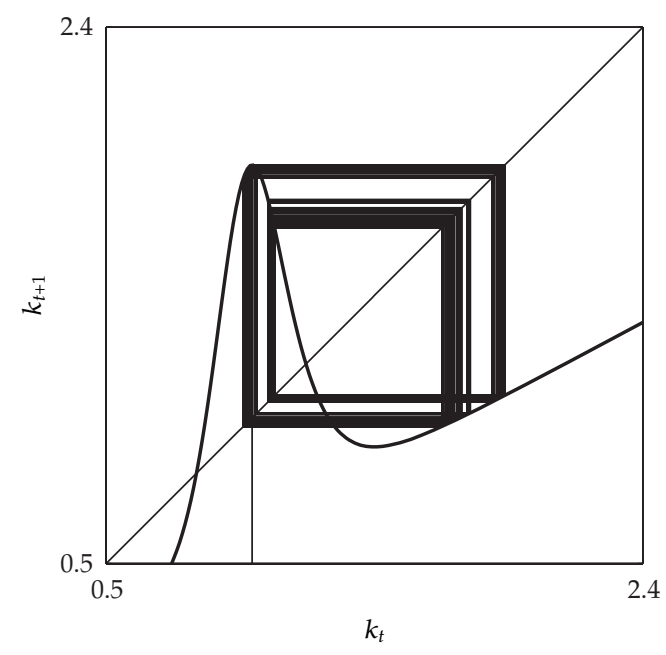

(a)

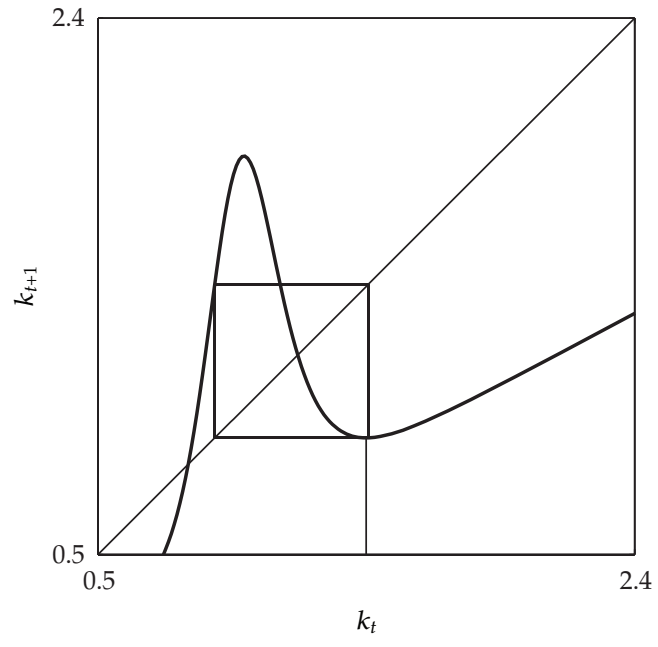

(b)

Figure 4: Coexisting attractors of $F$ for the parameter values considered in Figure 3 and $p=12$. (a) 4-pieces chaotic attractor for the initial condition $k_{M}$; (b) cycle-2 for the initial condition $k_{m}$.

(ii) If $F\left(k_{M}\right)>(<) k_{m}$ and $F\left(k_{m}\right)>(<) k_{M}$ then $k_{m} \in(\notin) S$ while $k_{M} \notin(\in) S$. This means that only the minimum (maximum) point belongs to the trapping set $S$ hence $F_{S}$ is unimodal. In such a case $A$ consists of a fixed point, an $m$-period cycle or a more complex attractor. The bifurcation structure eventually leading to complexity is the period-doubling bifurcation cascade (see Figure 3(b)).

(iii) If $F\left(k_{M}\right)>k_{m}$ and $F\left(k_{m}\right)<k_{M}$ then the two critical points belong to $F_{S}$; this means that $F$ is bimodal in $S$ (see Figure 3(c)) so that two coexisting attractors may be presented, namely, $A_{M}$ and $A_{m}$, where $A_{M}\left(A_{m}\right)$ is the set attracting the trajectory starting from the maximum (minimum) point. In such a case the attractor $A$ inside $S$ is given by $A=A_{M} \cup A_{m}$. About the coexistence of attractors in bimodal maps see Mira et al. [25].

In Figure 4 we show the two coexisting attractors $A_{m}$ and $A_{M}$ by choosing parameter values such that $F_{S}$ is bimodal. Observe that $A_{M}$ is a 4-pieces chaotic attractor while $A_{m}$ is a 2-period cycle.

Recall that we are considering the cases in which the unstable fixed point $k_{1}$ does not belong to the trapping set $S$, and consequently the basins of attraction of $A_{0}$ and of $A$ have a simple structure (simply connected basins), that is, $B\left(A_{0}\right)=\left[0, k_{1}\right)$ and $B(A)=\left(k_{1},+\infty\right)$.

In order to discuss the bifurcations leading to chaos we present some numerical simulations. Hence we fix the following parameter values: $\delta=0.2, \alpha=1, \beta=0.9, s_{w}=0.1, n=0.5$ and we let parameters $p$ and $\Delta s=s_{r}-s_{w}$ vary. In fact we are mostly interested in the role played by parameters $p$ and $\Delta s$ : the former informs about the elasticity of substitution (that decreases as $p$ increases), while the latter takes into account the difference between the two saving rates (once $s_{w}$ is fixed).

As we have discussed, the system becomes more and more complex as $p$ increases. This consideration is also supported by looking at Figure 3 showing that $F_{S}$ becomes bimodal as $p$ increases. In order to better understand the role of parameter $p$ that is strictly related to the elasticity of substitution in our model, we describe the sequence of bifurcations of $F$ 


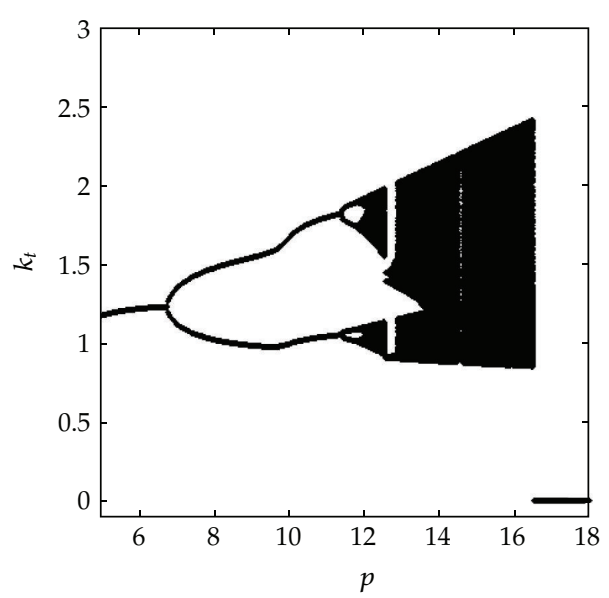

(a)

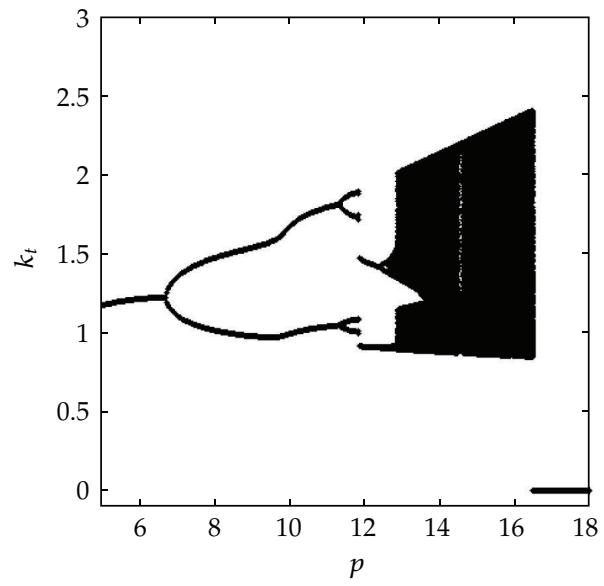

(b)

Figure 5: One dimensional bifurcation diagram of map $F$ w.r.t. $p$ for $s_{r}=0.7$. (a) $k_{0}=k_{M}$ and the attractor $A_{M}$ is presented; (b) $k_{0}=k_{m}$ and the attractor $A_{m}$ is presented.

w.r.t. $p$ and we show that multistability occurs (about multistability see, among others, Bischi et al. [26] and Sushko et al. [27]). The bifurcation diagrams in Figure 5 show how dynamics are increasingly complex if the elasticity of substitution between production factors declines (i.e., $p$ increases). In Figure 5(a) we depicted the attractor for $k_{0}=k_{M}$ while in Figure 5(b) the initial condition is given by the minimum point.

Obverve first that the trajectory converges to $k_{2}$ if $p<6.77$; if $p$ still increases a period doubling bifurcation occurs and a 2-period cycle appears. After such a bifurcation in Figure 5(a) we observe a sequence of period doubling bifurcations as the maximum point entered in $S$ at $p \simeq 10$, while in Figure 5(b) we observe a break in the bifurcation diagram at $p \simeq 12$ as also the minimum point entered in $S$, hence multistability can be observed for suitable values of the parameters. Observe that for $p<16.46$ condition $F\left(k_{m}\right)>k_{1}$ holds so that every initial condition with $k_{0}>k_{1}$ generates bounded trajectories converging to an attractor inside $S$. On the contrary, if $F\left(k_{m}\right)<k_{1}$ the unstable point $k_{1}$ belongs to $S$ and a global bifurcation occurs (we will explain such a case later). This kind of bifurcation requires an analysis of the global dynamical properties of the system, that is, an analysis which is not based on the linear approximation of the map.

The following statement summarizes our previous considerations.

Proposition 4.2. Assume the same hypotheses of Proposition 4.1. Then $A \subseteq S$ attracts all trajectories starting from $B(A)=\left(k_{1},+\infty\right)$ (it may consist of a fixed point, an n-period cycle or a strange attractor or it can be the union of two coexisting attractors $A_{M}$ and $A_{m}$ ) while $A_{0}=\{0\}$ attracts all trajectories starting from $B\left(A_{0}\right)=\left[0, k_{1}\right)$.

\subsection{Contact Bifurcations and Complex Basins}

As function $F$ is bimodal then it admits two critical points $k_{M}$ and $k_{m}$ such that $F\left(k_{M}\right)$ and $F\left(k_{m}\right)$ separates the set $\mathbb{R}_{+}$into two subset: $Z_{1}=\left[0, F\left(k_{m}\right)\right) \cup\left(F\left(k_{M}\right),+\infty\right)$ whose points have one rank-1 preimage and $Z_{3}=\left(F\left(k_{m}\right), F\left(k_{M}\right)\right)$ whose points have three rank-1 preimages 
(in the local maximum and minimum the two merging preimages $k_{m}$ and $k_{M}$ are located). As a consequence $F$ is a $Z_{1}-Z_{3}-Z_{1}$ non invertible map where $Z_{3}$ is the portion of $\mathbb{R}_{+}$bounded by the relative minimum value and the relative maximum value. Furthermore, as three fixed points are owned by $F$, then the origin is a locally stable fixed point, $k_{1}$ is a locally unstable fixed point while $k_{2}$ may be locally stable or unstable and one $(A)$ or two $\left(A_{M}\right.$ and $\left.A_{m}\right)$ more complex attractors may exist around $k_{2}$. As our map $F$ admits more then one attracting set, each with its own basin of attraction, we have to describe their changes as the parameter of the model vary. About the structure and the bifurcations related to the basins of attraction see Mira et al. [25].

In the previous subsection we described the structure of set $A$ when $F\left(k_{m}\right)>k_{1}$ and we showed that multistability due to the presence of two coexisting attractors occurs. Notice also that if $F\left(k_{m}\right)=k_{1}$ then a critical point of $F$ is pre-periodic. Thus no attracting cycles exist since the basin of attraction of these cycles cannot contain the critical point $k_{1}$, providing the evidence of the existence of parameter values such that the map is chaotic.

A completely different situation appears if $F\left(k_{m}\right)$ crosses $k_{1}$ as a contact bifurcation occurs. Such a bifurcation happens if a parameter variation causes a crossing between a basin boundary and a critical set so that a portion of a basin enters in a region where an higher number of inverses is defined, then new components of the basin suddenly appear after the contact (see Bischi et al. [28]). Obviously trajectories starting from $B\left(A_{0}\right)$ converge to the origin (immediate basin), hence if the economy starts from a low level of economic growth it will fall in the poverty trap. On the other hand, if some parameters change this causes the minimum value $F\left(k_{m}\right)$ to cross $k_{1}$, then the portion $\left(F\left(k_{m}\right), k_{1}\right)$ enters in $Z_{3}$ so that new preimages appear. In fact $k_{1}$ has two new preimages given by $\left(k_{1}^{a}\right)_{-1}<k_{m}$ and $\left(k_{1}^{b}\right)_{-1}>k_{m}$ and consequently initial conditions belonging to $B_{-1}=\left(\left(k_{1}^{a}\right)_{-1},\left(k_{1}^{b}\right)_{-1}\right)$ also generate trajectories converging to zero as $B_{-1}$ is mapped into set $B\left(A_{0}\right)$ after one iteration.

Let $F^{2}\left(k_{M}\right)<k_{1}$, so that the second iterate of the maximum point does not belong to the immediate basin of the origin $B\left(A_{0}\right)$, then the previous procedure can be repeated while considering the preimages of rank-2 of the unstable fixed point $k_{1}$. Again, let $\left(k_{1}^{a}\right)_{-2}$ and $\left(k_{1}^{b}\right)_{-2}$ be the preimages of $\left(k_{1}^{a}\right)_{-1}$ and $\left(k_{1}^{b}\right)_{-1}$, then initial conditions belonging to the set $B_{-2}=\left(\left(k_{1}^{a}\right)_{-2},\left(k_{1}^{b}\right)_{-2}\right)$ generate trajectories converging to $B\left(A_{0}\right)$ after two iterations. The story repeates and a set of non-connected portion is created, so that the contact between the critical set and the basin boundary marks the transition between simple connected to non-connected basins. Finally the basin of attraction of the origin is given by

$$
B(0)=B\left(A_{0}\right) \cup_{i \geq 1}\left(\left(k_{1}^{a}\right)_{-i^{\prime}}\left(k_{1}^{b}\right)_{-i}\right) .
$$

In such a case an economic policy trying to push up the investment does not guarantees the exit from the poverty trap (see Figure 6(a)).

In Figure 7 we present two trajectories converging to different attractors for close initial conditions: in Figure 7(a) $k_{0}=1.26$ belongs to the basin of $A$ and $A$ consists in a very high period cycle or in a chaotic set; in Figure $7(b)$ the initial condition is $k_{0}=1.27$ belonging to the basin of the origin. In fact in such a case being $F\left(k_{m}\right)<k_{1}$ the basin structure is complex as it consists of infinitely many non-connected sets.

Finally, at $F^{2}\left(k_{M}\right)=k_{1}$ a final bifurcation occurs such that the maximum point is attracted by the unstable fixed point $k_{1}$ and the map is chaotic (see Devaney [29]). After such a final bifurcation $F^{2}\left(k_{M}\right)<k_{1}$ and $A$ is a Cantor set (the origin attracts almost all trajectories, see Figure 6(b)). 


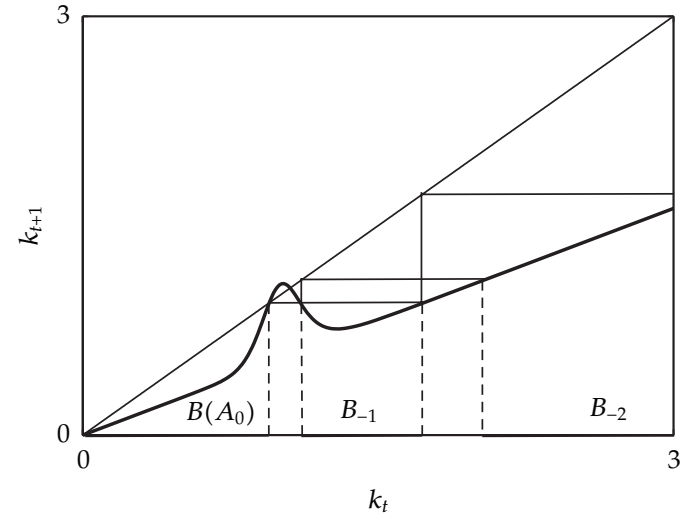

(a)

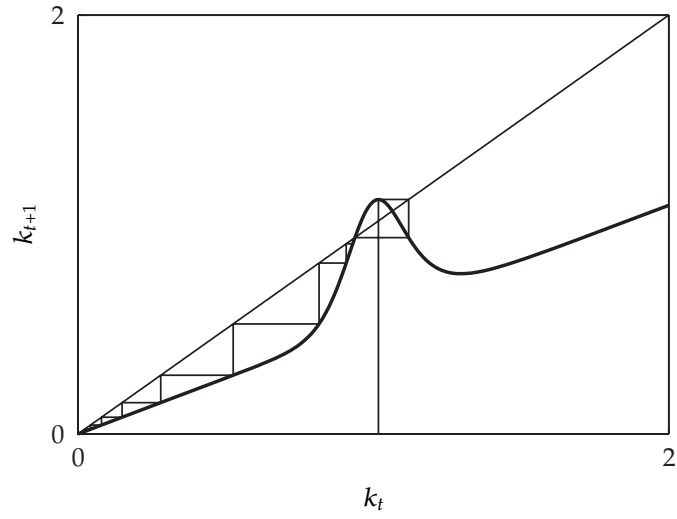

(b)

Figure 6: (a) Unconnected basin of attraction of the origin for the following parameter values: $\beta=0.9$, $s_{w}=0.1, s_{r}=0.7, p=15, \delta=0.2, n=0.5$, and $\alpha=0.32$. (b) After the final bifurcation the origin attracts the trajectory starting from the maximum point for $\alpha=0.34$.

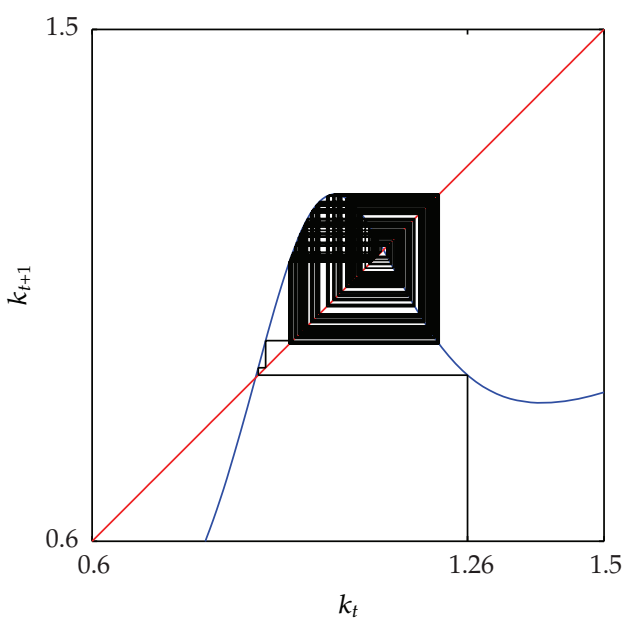

(a)

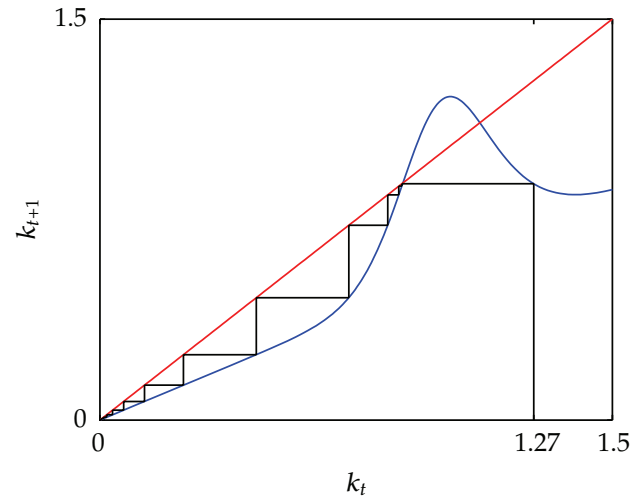

(b)

Figure 7: K-L staircase diagram for the following parameter values: $\delta=0.2, \alpha=0.4, \beta=0.9, n=0.5$, $s_{w}=0.1, s_{r}=0.9$, and $p=11$. (a) $k_{0}=1.26$ generates a trajectory converging to $A$ consisting in a complex set; (b) $k_{0}=1.27$ generates a trajectory converging to $A_{0}$.

\subsection{Numerical Simulations}

The joint analysis of the map w.r.t. $p$ and $\Delta s$ explains how the elasticity of substitution in the nonconcave production function affects the final long run dynamics of the growth model for different values of the difference between the two saving rates. In Figure 8 we present two cycle cartograms showing a two parametric bifurcation diagram qualitatively: each color represents a long-run dynamic behaviour for a given point in the parameter plane $(\Delta s, p)$ and for the initial conditions $k_{0}=k_{M}$ and $k_{0}=k_{m}$, respectively. A large diversity of cycles of different order is exhibited. The red region represents the parameter values for which 


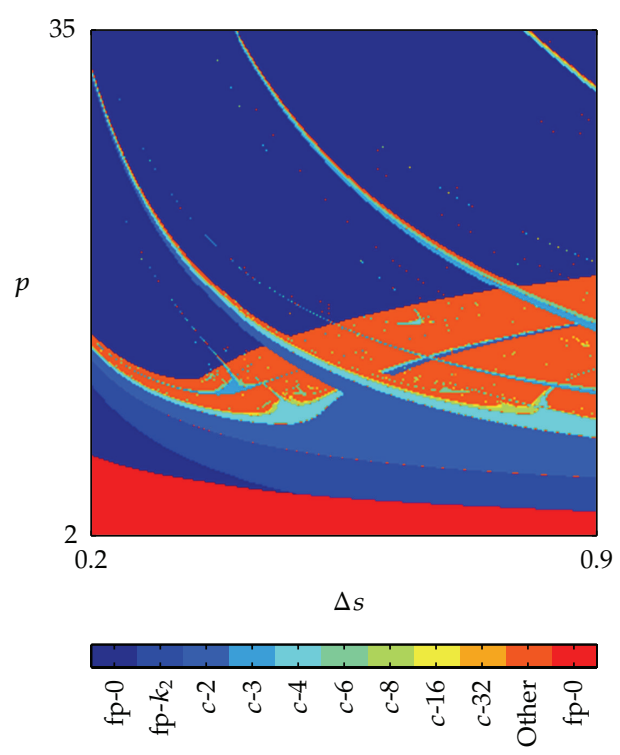

(a)

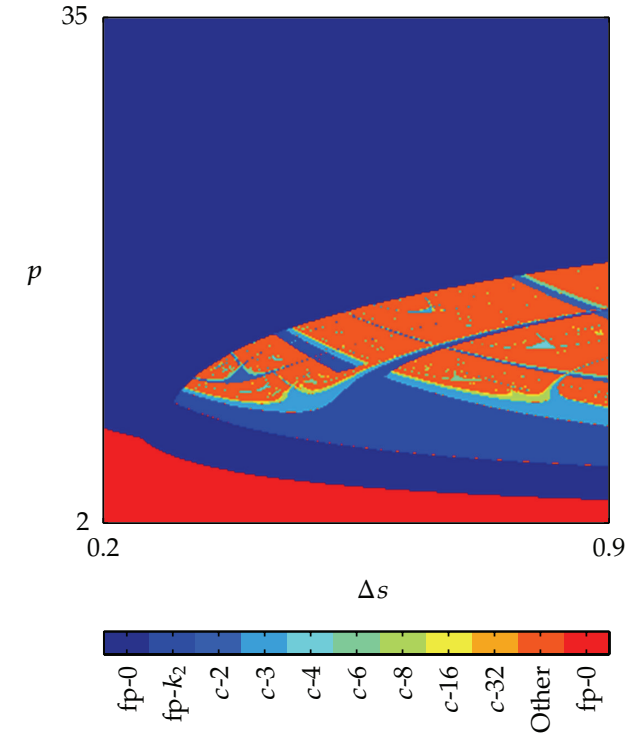

(b)

Figure 8: Two dimensional bifurcation diagrams of map $F$ in the plain $(\Delta s, p)$ for the following parameter values: $\delta=0.2, \alpha=1, \beta=0.9, n=0.5, s_{w}=0.1$; (a) the initial condition is $k_{M}$; (b) the initial condition is $k_{m}$.

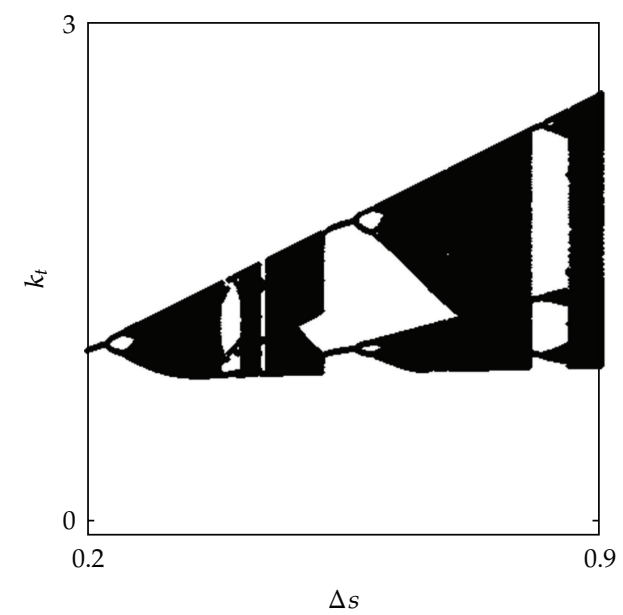

(a)

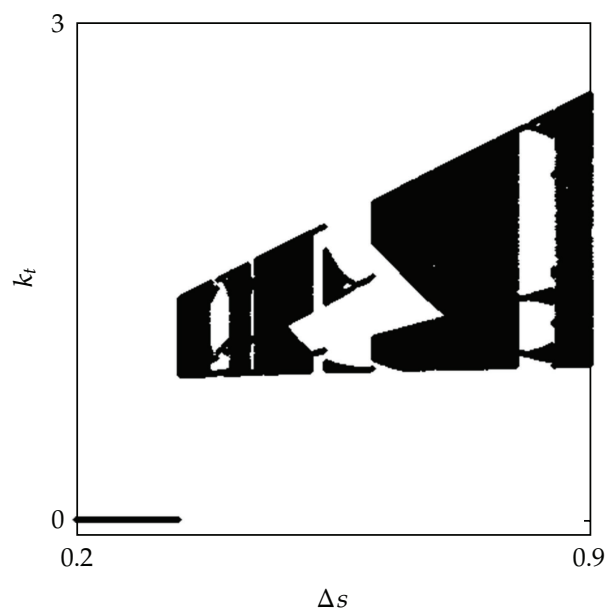

(b)

Figure 9: Bifurcation diagrams w.r.t. $\Delta s$ for the following parameter values: $\delta=0.2, \alpha=1, \beta=0.9, n=0.5$, $s_{w}=0.1$, and $p=12$; (a) the initial condition is $k_{M}$; (b) the initial condition is $k_{m}$.

the function is monotonic and the origin is globally stable; this situation occurs for small values of $p$. Also in the dark blue region the system fails to converge to the origin (poverty trap), anyway we have entered into the region of bimodality.

Firstly observe that once $p$ is fixed at an intermediate level, the final dynamics of the system becomes more complex as the difference between the two saving rates increases. In Figure 9 we present two bifurcation diagrams w.r.t. $\Delta s$ showing how dynamics is increasingly 


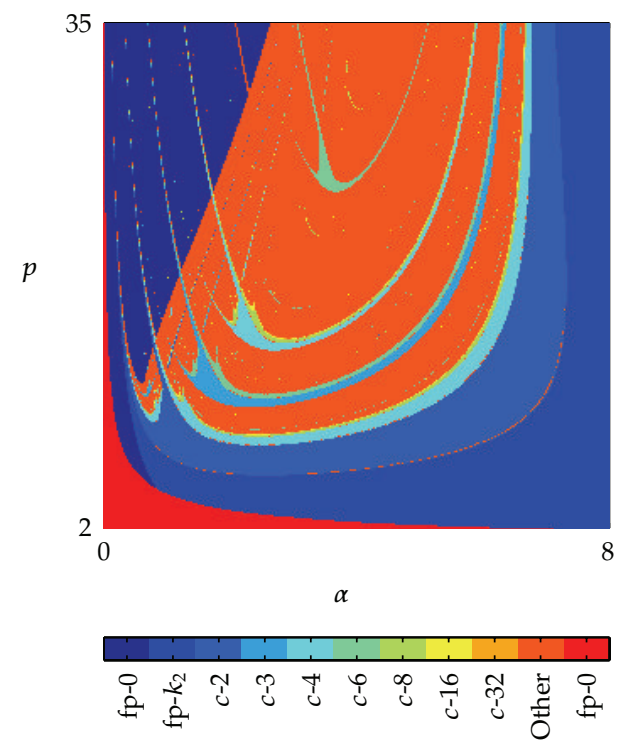

(a)

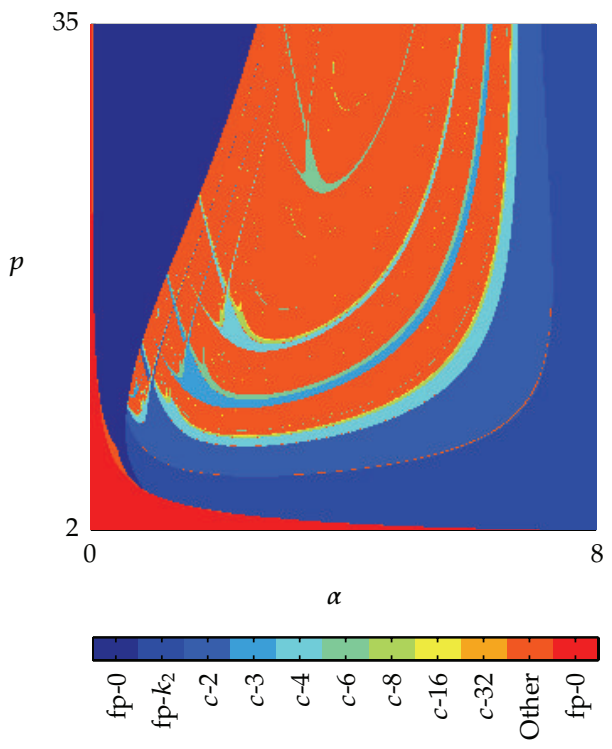

(b)

Figure 10: Two dimensional bifurcation diagrams of map $F$ in the plain $(\alpha, p)$ for the following parameter values: $\delta=0.2, s_{r}=0.7, s_{w}=0.1, \beta=0.9, n=0.5$; (a) the initial condition is $k_{M}$; (b) the initial condition is $k_{m}$.

complex if the difference between the two saving rates is large enough. This result confirms the one reached in previous studies where CES or VES production functions were considered (see Brianzoni et al. [11, 13, 14]).

Secondly, parameter $p$ is the one related to the nonconstant elasticity of substitution between production factors: it decreases as $p$ increases. Observe that once $\Delta s$ is fixed complex dynamics can be observed in our model for intermediate values of $p$, till a contact bifurcation occurs, providing that in order for fluctuations to arise the elasticity of substitution between production factors must not be too small. Notice that, differently from previous studies (see Brianzoni et al. $[11,13,14])$ after the contact bifurcation occurring at an high value of $p$ the system will converge again to the poverty trap.

In Figure 10 we consider the role of $p$ and that of $\alpha$. Both parameters are related to the properties of the production function as $p$ informs about the elasticity of substitution while $\alpha$ explains the upper bound of the production function. Furthermore, we proved that both $p$ and $\alpha$ must not be too small for $F$ being bimodal and multiple equilibria being owned. In fact one can observe that if the two parameters do not tend to their lower bound the complex behaviour is possible while if $(\alpha, p) \rightarrow(0,2)$ then $F$ is strictly increasing.

In Figure 11 we present the bifurcation diagrams w.r.t. $\alpha$ for different values of $p$. For $p=6$ we observe a period halving forming a closed loop-like structure called a primary bubble. In all the cases a period doubling and a period halving cascade is observed. Complex features are observed for intermediate levels of $\alpha$; in fact, as proved, $\alpha$ must be great enough for $F$ to have three fixed points and low enough for the final bifurcation to have not occurred. 


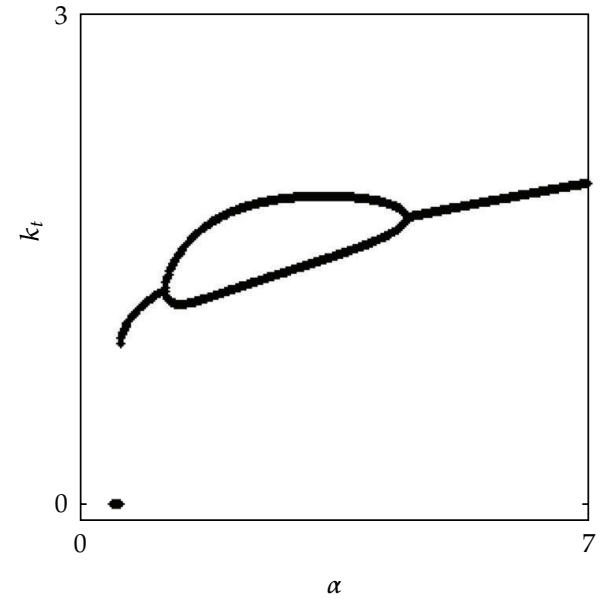

(a)

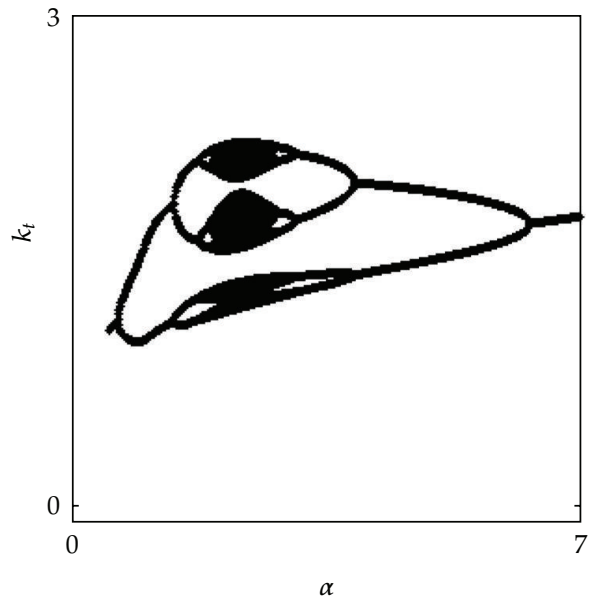

(c)

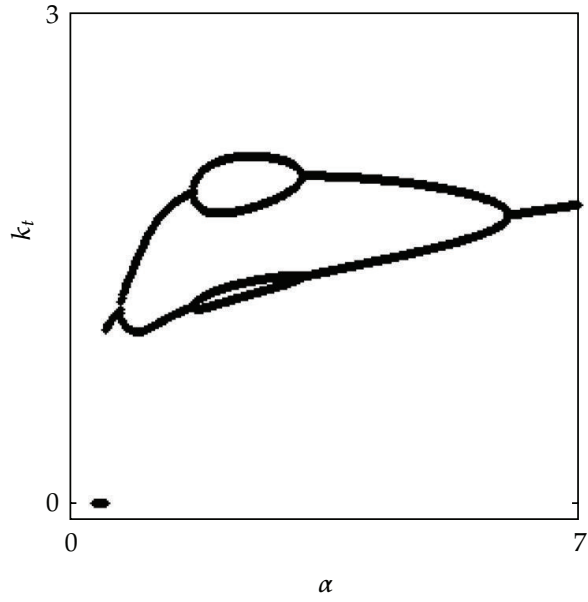

(b)

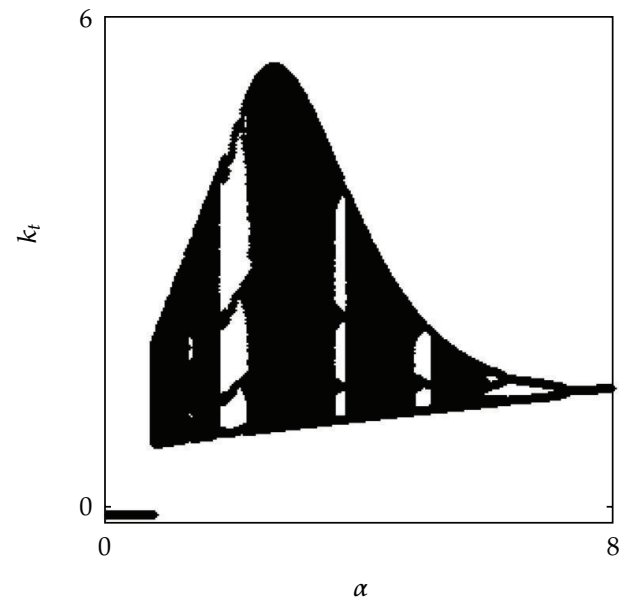

(d)

Figure 11: Bifurcation diagrams w.r.t. $\alpha$ for different values of $p$ and for the following parameter values: $\delta=0.2, s_{r}=0.8, s_{w}=0.1, \beta=0.9, n=0.5$ and the initial condition $k_{0}=k_{m}$; (a) $p=6$; (b) $p=7.5$, (c) $p=8$, (d) $p=15$.

\section{Conclusions}

In this work we considered a Solow-Swan growth model in discrete time with differential savings between workers and shareholders as in Böhm and Kaas [10], while using a convexconcave production function as in Capasso et al. [23].

The study conducted represents a further step in the knowledge of the role played by the elasticity of substitution and the difference between saving rates in generating cycles or complex dynamics in simple neoclassical growth models. In fact, the results herewith obtained can be compared with the ones reached in Brianzoni et al. [11, 13] using CES production function and that obtained in Brianzoni et al. [14] using the VES production function in the form given by Revankar [15]. As a matter of fact, also the nonconcave production 
function we introduced has a variable elasticity of substitution as the VES, anyway, differently from the VES it does not satisfy the positiveness of the second derivative.

Our study aims at confirming that the elasticity of substitution between production factors plays a crucial role in economic growth theory (see Solow [1]). In fact as in all the studies above mentioned, cycles or complex dynamics can emerge if shareholders save more than workers and the elasticity of substitution between production factors is low enough. Anyway, a new feature is due to the fact that, being $F$ a bimodal function having coexisting attractors, then a final bifurcation occurs at which complicated dynamics is ruled out for very low values such elasticity.

As with the CES (and differently from the VES) unbounded endogenous growth cannot be observed as the coexisting attractors of the system consist of fixed points or compact sets.

Anyway, differently from the other cases studied, with nonconcave production function the origin is always a locally stable fixed point so that the system may converge to the poverty trap.

Finally, the existence of such an attractor for our bimodal map implies multistability and basins complexity.

\section{Acknowledgment}

The authors acknowledge financial support by Minister of Education, Italy (MIUR) through the 2009 Research Project of National Relevance (PRIN) on Structural Change led by Neri Salvadori.

\section{References}

[1] R. M. Solow, "A contribution to the theory of economic growth," Quarterly Journal of Economics, vol. 70, pp. 65-94, 1956.

[2] T. W. Swan, "Economic growth and capital accumulation," Economic Record, vol. 32, pp. 334-361, 1956.

[3] R. Klump and H. Preissler, "CES production functions and economic growth," Scandinavian Journal of Economics, vol. 102, no. 1, pp. 41-56, 2000.

[4] W. H. Masanjala and C. Papageorgiou, "The solow model with ces technology: nonlinearities and parameter heterogeneity," Journal of Applied Econometrics, vol. 19, no. 2, pp. 171-201, 2004.

[5] R. Klump and O. de La Grandville, "Economic growth and the elasticity of substitution: two theorems and some suggestions," American Economic Review, vol. 90, no. 1, pp. 282-291, 2000.

[6] N. Kaldor, "Alternative theories of distribution," Review of Economic Studies, vol. 23, pp. 83-100, 1956.

[7] N. Kaldor, “A model of economic growth," Economic Journal, vol. 67, pp. 591-624, 1957.

[8] L. L. Pasinetti, "Rate of profit and income distribution in relation to the rate of economic growth," Review of Economic Studies, vol. 29, pp. 267-279, 1962.

[9] P. A. Samuelson and F. Modigliani, "The Pasinetti paradox in neoclassical and more general models," Review of Economic Studies, vol. 33, pp. 269-301, 1966.

[10] V. Böhm and L. Kaas, "Differential savings, factor shares, and endogenous growth cycles," Journal of Economic Dynamics and Control, vol. 24, no. 5-7, pp. 965-980, 2000.

[11] S. Brianzoni, C. Mammana, and E. Michetti, "Complex dynamics in the neoclassical growth model with differential savings and non-constant labor force growth," Studies in Nonlinear Dynamics and Econometrics, vol. 11, no. 3, article 3, 17 pages, 2007.

[12] S. Brianzoni, C. Mammana, and E. Michetti, "Global attractor in solow growth model with differential savings and endogenic labor force growth," AMSE Periodicals, Modelling Measurement and Control D, vol. 29, no. 2, pp. 19-37, 2008.

[13] S. Brianzoni, C. Mammana, and E. Michetti, "Nonlinear dynamics in a business-cycle model with logistic population growth," Chaos, Solitons and Fractals, vol. 40, no. 2, pp. 717-730, 2009. 
[14] S. Brianzoni, C. Mammana, and E. Michetti, "Variable elasticity of substituition in a discrete time Solow-Swan growth model with differential saving," Chaos, Solitons and Fractals, vol. 45, pp. 98-108, 2012.

[15] N. S. Revankar, "A class of variable elasticity of substitution production function," Econometrica, vol. 39, pp. 61-71, 1971.

[16] G. Karagiannis, T. Palivos, and C. Papageorgiou, "Variable elasticity of substitution and economic growth," in New Trends in Macroeconomics, D. Claude and C. Kyrtsou, Eds., pp. 21-37, Springer, Berlin, Germany, 2005.

[17] R. A. Becker, "Equilibrium dynamics with many agents," in Handbook of Optimal Growth Theory, R. A. Dana, C. le Van, T. Mitra, and K. Nihimura, Eds., Springer, Berlin, Germany, 2006.

[18] C. W. Clark, "Economically optimal policies for the utilization of biologically renewable resources," Mathematical Biosciences, vol. 12, pp. 245-260, 1971.

[19] A. K. Skiba, "Optimal growth with a convex-concave production function," Econometrica, vol. 46, no. 3, pp. 527-539, 1978.

[20] T. Kamihigashi and S. Roy, "Dynamic optimization with a nonsmooth, nonconvex technology: the case of a linear objective function," Economic Theory, vol. 29, no. 2, pp. 325-340, 2006.

[21] T. Kamihigashi and S. Roy, "A nonsmooth, nonconvex model of optimal growth," Journal of Economic Theory, vol. 132, no. 1, pp. 435-460, 2007.

[22] M. Majumdar and T. Mitra, "Dynamic optimization with a nonconvex technology: the case of a linear objective function," Review of Economic Studies, vol. 50, no. 1, pp. 143-151, 1983.

[23] V. Capasso, R. Engbers, and D. La Torre, "On a spatial Solow model with technological diffusion and nonconcave production function," Nonlinear Analysis: Real World Applications, vol. 11, no. 5, pp. 3858-3876, 2010.

[24] E. Liz, "Complex dynamics of survival and extinction in simple population models with harvesting," Theoretical Ecology, vol. 3, no. 4, pp. 209-221, 2010.

[25] C. Mira, L. Gardini, A. Barugola, and J.-C. Cathala, Chaotic Dynamics in Two-Dimensional Noninvertible Maps, vol. 20, World Scientific Publishing, Singapore, 1996.

[26] G. I. Bischi, C. Mammana, and L. Gardini, "Multistability and cyclic attractors in duopoly games," Chaos, Solitons and Fractals, vol. 11, no. 4, pp. 543-564, 2000.

[27] I. Sushko, A. Agliari, and L. Gardini, "Bistability and border-collision bifurcations for a family of unimodal piecewise smooth maps," Discrete and Continuous Dynamical Systems B, vol. 5, no. 3, pp. 881-897, 2005.

[28] G.-I. Bischi, L. Gardini, and C. Mira, "Contact bifurcations related to critical sets and focal points in iterated maps of the plane," in Proceedings of the International Workshop Future Direction in Difference Equation, vol. 69, pp. 15-50, Vigo, Spain, June 2011.

[29] R. L. Devaney, An Introduction to Chaotic Dynamical Systems, Benjamin, Menlo Park, Calif, USA, 1986. 


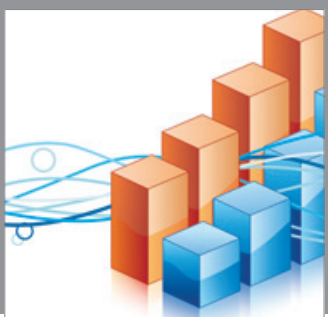

Advances in

Operations Research

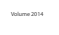

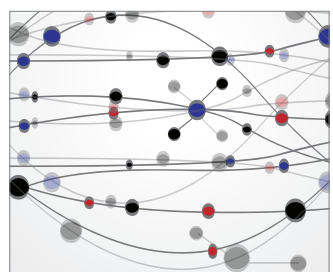

\section{The Scientific} World Journal
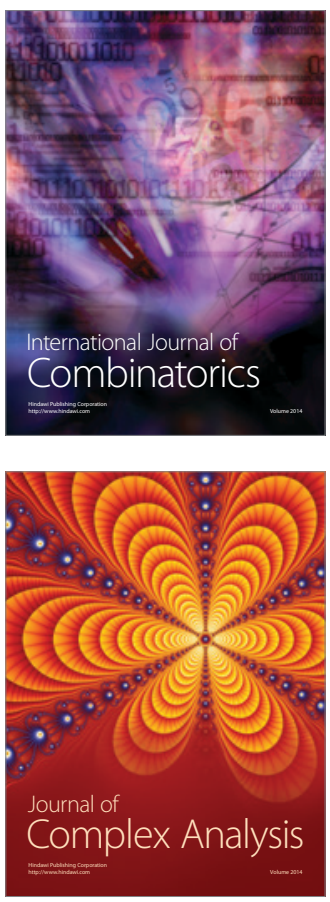

International Journal of

Mathematics and

Mathematical

Sciences
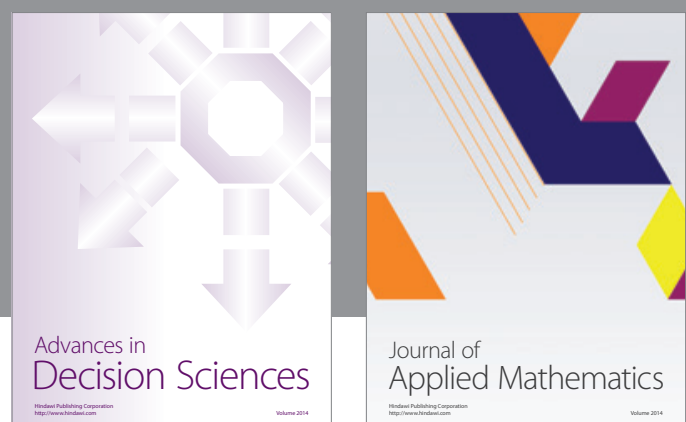

Journal of

Applied Mathematics
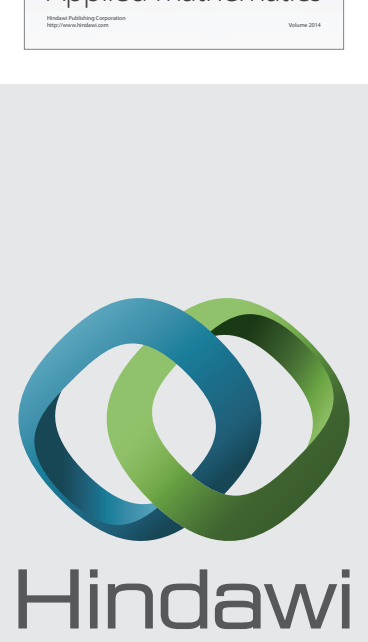

Submit your manuscripts at http://www.hindawi.com
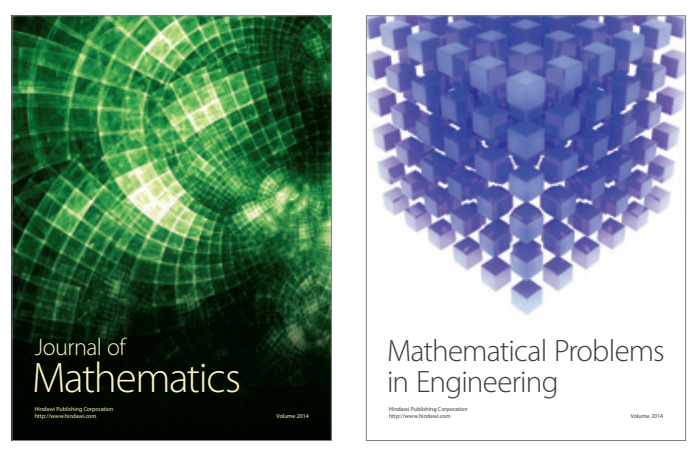

Mathematical Problems in Engineering
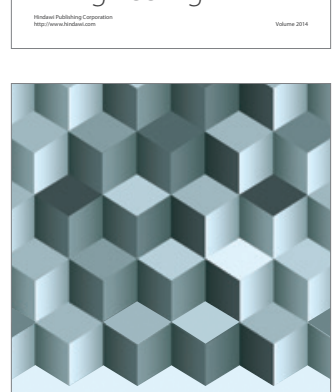

Journal of

Function Spaces
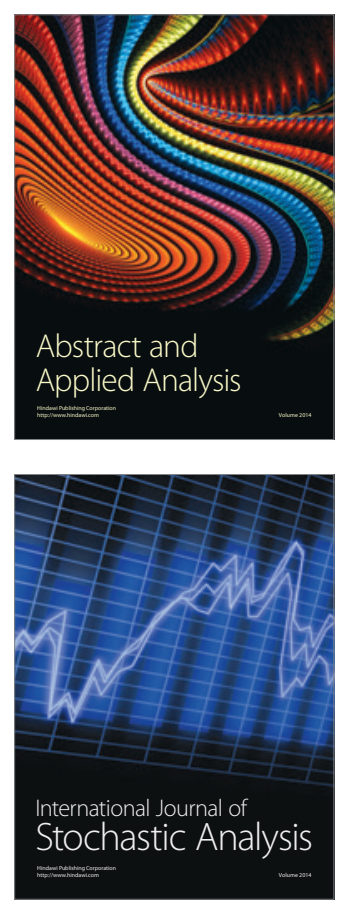

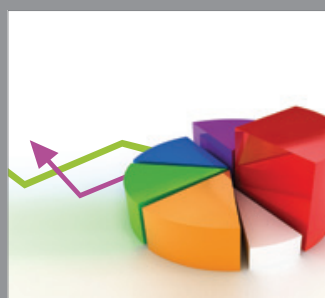

ournal of

Probability and Statistics

Promensencen
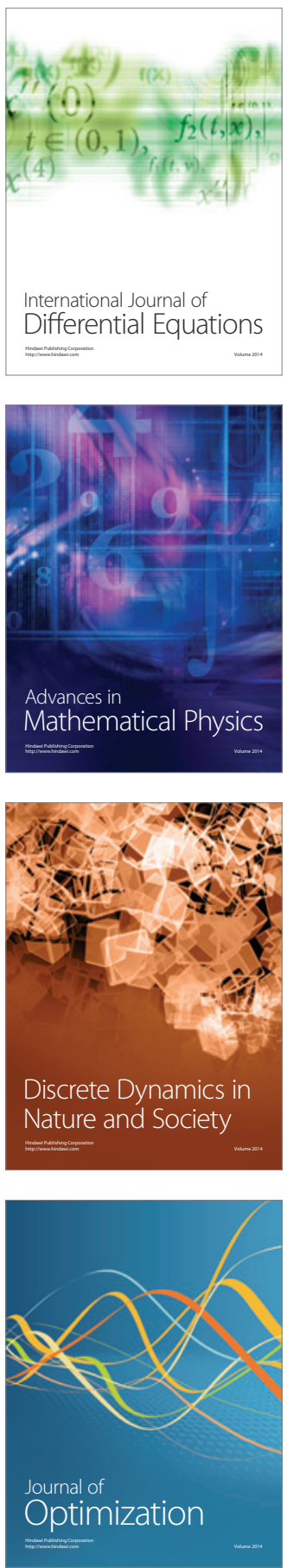